\title{
Troponin as a biomarker for mortality in stable COPD
}

\author{
Ulf Nilsson $\mathbb{1}^{1}$ and Lowie E.G.W. Vanfleteren $\mathbb{1}^{2,3}$
}

Affiliations: ${ }^{1}$ Dept of Public Health and Clinical Medicine, Section of Medicine, Umeå University, Umeå, Sweden. ${ }^{2}$ COPD Center, Dept of Respiratory Medicine and Allergology, Sahlgrenska University Hospital, Gothenburg, Sweden. ${ }^{3}$ COPD Center, Dept of Internal Medicine and Clinical Nutrition, Institute of Medicine, Sahlgrenska Academy, University of Gothenburg, Gothenburg, Sweden.

Correspondence: Ulf Nilsson, Umeå University Hospital, B41, 90185 Umeå, Sweden. E-mail: ulf.nilssondumu.SE

@ERSpublications

High sensitive cardiac troponins are important predictive cardiovascular biomarkers for all-cause mortality in COPD. Underlying causes to elevated troponin are still unclear, but elevated troponin might identify COPD phenotypes suitable for CVD prevention. http://bit.ly/35EKAqW

Cite this article as: Nilsson U, Vanfleteren LEGW. Troponin as a biomarker for mortality in stable COPD. Eur Respir J 2020; 55: 1902447 [https://doi.org/10.1183/13993003.02447-2019].

COPD is a leading cause of death in the world. COPD represents the pulmonary component of multimorbidity, often with cardiovascular disease (CVD) as the most prevalent, and possibly the most clinically relevant [1]. Indeed, cardiovascular diseases affect quality of life and healthcare costs, and are a major cause of death in COPD [2, 3]. The Global Initiative for Chronic Obstructive Lung Disease (GOLD) initiative recognises CVD as the most important disease coexisting with COPD and suggests it should be routinely looked for, suggesting the use of a CVD risk calculator [4].

Although plenty of well conducted research has been done in the past decades related to the pathophysiological connections between COPD and CVD, these are still not fully understood [5]. Smoking and inactivity are common risk factors, and the metabolic syndrome is commonly seen in COPD [6]. However, multiple variables in COPD are likely to contribute to the complexity of coexistence of cardiovascular disease and COPD. For example, enhanced systemic inflammatory response, hypoxaemia, sleep disturbances, exacerbations and lung hyperinflation, have all been put forward as contributing factors connecting both diseases.

Troponins can be found in the myocardium, as well as in skeletal muscles. The cardiac specific troponins (cTn) $\mathrm{T}$ and I are considered to be important biomarkers for the diagnosis of myocardial infarction. In addition, cTn may provide a useful screening tool to identify patients at high and low cardiac risk. In this context, much lower troponin cut-off levels than the current thresholds for myocardial infarction are employed $[7,8]$.

In this issue of the European Respiratory Journal, WASCHKI et al. [9] report results from the German COSYCONET cohort, which includes 2741 patients with a clinical diagnosis of COPD, mainly GOLD stage 2 and 3 . The main aim was to investigate the predictive value of high-sensitivity cardiac troponin I (hs-cTnI) on all-cause mortality among the 2020 COPD patients in COSYCONET with detectable hs-cTnI levels during a 3-year follow-up, and also to identify determinants of elevated hs-cTnI levels. In the current study, hs-cTnI was analysed in quartiles, as a continuous log-transformed variable and as a cut-off of $>6 \mathrm{ng} \cdot \mathrm{L}^{-1}$, well below the 99th percentile, the clinical threshold for diagnosis of myocardial infarction recommended by the European Society of Cardiology [7]. 
When analysing quartiles of hs-cTnI, the authors showed that patients in the highest quartile had an increased mortality risk compared to the lowest quartiles. When analysing mortality risk using multivariate regression models, increased hs-cTnI, as continuous variable as well as with a cut-off at $>6 \mathrm{ng} \cdot \mathrm{L}^{-1}$, were strong independent predictors for all-cause mortality in COPD. The authors conclude that circulating hs-cTnI concentrations might be a useful tool to identify patients at high risk across the whole COPD severity spectrum early in the disease process, thus enabling closer monitoring and treatment of these patients.

The main findings of this study are interesting and of potential importance for the identification and management of COPD patients at risk of developing cardiovascular diseases. The results add to the growing body of evidence implying that patients with a physician-diagnosed COPD and elevated cardiac troponins have an increased mortality risk during up to 36 months follow-up [10, 11]. But what does an increase in circulating troponin represent in subjects with COPD? One likely explanation for elevated troponin is subclinical CVD, and subjects with COPD may also have a higher prevalence of subclinical CVD compared to subjects without COPD [12]. Other possible causes of elevated levels of circulating cardiac troponin in COPD could be increased levels of hypoxaemia or left ventricular dysfunction, as indicated in this paper, and/or effects related to the enhanced systemic inflammatory response as seen in a proportion of COPD patients. However, elevated cardiac troponin is not unique for COPD. Detectable levels of hs-cTnT, up to $13.9 \mathrm{ng} \cdot \mathrm{L}^{-1}$, has been found in nearly $50 \%$ of subjects with ideal cardiovascular health in a population-based study [13].

Indeed, little is known about the factors that determine the onset of CVD and even less is known about the relationship between troponin and the pathogenesis of COPD. A low lung function in early adulthood has been associated with approximately a 10 year earlier development of comorbid disease, including CVD [14]. One could hypothesise that high levels of a specific marker of cardiac risk early in life, could specify a population at risk for multi-organ pathology, including lung damage related to exposure.

Tobacco is an important shared risk factor for both COPD and CVD. In this paper, tobacco, categorised as current smoking, showed only univariate association with higher levels of hs-cTnI. A higher cumulative tobacco consumption, assessed as pack-years, has previously been associated with elevated troponin [15]. The relationship between tobacco and CVD in COPD is not surprising, but could a high tobacco consumption together with elevated $\mathrm{cTn}$ in a general population also work as a risk marker for future development of COPD?

Pharmacological interventions on both the cardiovascular and COPD side have been evaluated against outcomes that might be influenced by CVD-COPD interactions, but were consistently negative. For example, in the large SUMMIT study, ICS-LABA compared to placebo failed to reduce mortality in patients with CVD or increased cardiovascular risk [11]. In addition, both statins [16] and beta-blockers [17] were unsuccessful in reducing COPD related outcomes, such as exacerbations, in subjects who did not have a CVD-related indication for that therapy. Biomarkers like troponin could potentially help to identify subjects and personalise therapy for a phenotype with increased cardiovascular risk.

Exacerbations have been associated with cardiovascular risk in multiple ways [18] and increased troponin levels are commonly seen during exacerbations. In the current study, a history of severe exacerbations (requiring hospitalisation) is associated with higher hs-cTnI levels measured even at stable phase, independent of prevalent CVD, age, sex and smoking status. However, hs-cTnI did not have any predictive value for the prediction of future exacerbations of COPD or exacerbation-related hospitalisations of COPD. This is an intriguing result, given the fact that the historical association is present, and hs-cTnI is able to predict all-cause mortality. A history of exacerbations is a strong predictor of future exacerbation risk [19] and severe exacerbations have been connected to a ten-fold increased risk in cardiovascular incidence in a post hoc analysis of SUMMIT [20]. In addition, the lack of specificity of the definition of exacerbations has been criticised, as a flare-up of symptoms can be related to comorbid disease. These findings emphasise the urgent need to better characterise exacerbations of COPD. Unfortunately, the current study does not provide information on cardiovascular incidence or cause-specific mortality.

In the current cohort, hs-cTnI was a better predictor of mortality than $\mathrm{N}$-terminal pro-brain natriuretic peptide (NT-proBNP). NT-proBNP is a biomarker used in the diagnosis of congestive heart failure, with levels $<300 \mathrm{pg} \cdot \mathrm{mL}^{-1}$ considered to have a $99 \%$ negative predictive value. In the current study, elevated NT-proBNP, as well as right, but not left ventricular dysfunction, correlated with hs-cTnI $>6 \mathrm{ug} \cdot \mathrm{L}^{-1}$, but hs-cTnI was still a better predictor for mortality than NT-proBNP in the current study population. Nevertheless, NT-proBNP has previously been shown to be an independent predictor for future mortality in general populations as well as in selected patient groups, including COPD [21]. Interestingly, in the Nord-Trøndelag Health Study (HUNT) cohort, ROMUNDSTAD et al. [22] reported previously in the ERJ a positive association between microalbuminuria and all-cause mortality in subject with COPD. Microalbuminuria has been consistently associated with cardiovascular events [23]. 
Now more and more biomarkers known to be associated with adverse cardiovascular outcomes have been shown to be successful independent predictors of mortality in COPD populations. The question raises how they relate to each other, how they could be combined, and which aspects and phenotypes of COPD they relate to. Do these biomarkers identify a population to focus on for earlier and aggressive primary CVD prevention in COPD? We have to keep in mind that such studies have reported a greater degree of side-effects and adverse events [17]. Careful studies are needed to clarify the mechanism by which low levels of circulating troponin are released in general and in patients with COPD in particular, and how management strategies may influence ultimate outcomes.

In summary, the presence of troponin in patients with stable COPD is associated with all-cause mortality in the COSYCONET cohort, as published in this issue of the ERJ. Troponin levels are associated with a history of severe exacerbations, but do not predict future exacerbations. Carefully obtained longitudinal data of troponin in subjects with COPD and its relationship with other biomarkers, CVD incidence, incidence of better characterised exacerbations, specific COPD phenotypes, and cause-specific mortality are needed. Due to the vast under-diagnosis of COPD patients, more population-based studies are needed. Population-based studies identify a larger proportion of subjects with mild airway obstruction compared to patient recruited cohorts [24], and may be able to cast further light on the early onset of cardiovascular comorbidity in the earliest stages of COPD. Finally, markers like troponin that identify COPD patients at risk, could be considered for well-designed interventional trials that investigate effects of (cardiovascular) drugs in COPD.

Conflict of interest: U. Nilsson reports personal fees for lectures from Boehringer Ingelheim, outside the submitted work. L.E.G.W. Vanfleteren reports grants and personal fees from AstraZeneca, personal fees from Novartis, GSK, Chiesi, Menarini, Pulmonx and Boehringer, grants from Fisher and Paykel, outside the submitted work.

\section{References}

1 Putcha N, Drummond MB, Wise RA, et al. Comorbidities and chronic obstructive pulmonary disease: prevalence, influence on outcomes, and management. Semin Respir Crit Care Med 2015; 36: 575-591.

2 Berry CE, Wise RA. Mortality in COPD: causes, risk factors, and prevention. COPD 2010; 7: 375-382.

3 Zhang J, Rutten FH, Cramer MJ, et al. The importance of cardiovascular disease for mortality in patients with COPD: a prognostic cohort study. Fam Pract 2011; 28: 474-481.

4 Global Initiative for Chronic Obstructive Lung Disease. Global Strategy for the Diagnosis, Management, and Prevention of Chronic Obstructive Pulmonary Disease. https://goldcopd.org/wp-content/uploads/2019/12/GOLD2020-FINAL-ver1.2-03Dec19_WMV.pdf Date last accessed: 16 December, 2019.

5 Maclay JD, MacNee W. Cardiovascular disease in COPD: mechanisms. Chest 2013; 143: 798-807.

6 Cebron Lipovec N, Beijers RJ, van den Borst B, et al. The prevalence of metabolic syndrome in chronic obstructive pulmonary disease: a systematic review. COPD 2016; 13: 399-406.

7 Ibanez B, James S, Agewall S, et al. 2017 ESC Guidelines for the management of acute myocardial infarction in patients presenting with ST-segment elevation: The Task Force for the management of acute myocardial infarction in patients presenting with ST-segment elevation of the European Society of Cardiology (ESC). Eur Heart J 2018; 39: 119-177.

8 Chapman AR, Lee KK, McAllister DA, et al. Association of high-sensitivity cardiac troponin I concentration with cardiac outcomes in patients with suspected acute coronary syndrome. JAMA 2017; 318: 1913-1924.

9 Waschki B, Alter P, Zeller T, et al. High-sensitivity troponin I and all-cause mortality in patients with stable COPD: an analysis of the COSYCONET study. Eur Respir J 2020; 55: 1901314

10 Neukamm A, Einvik G, Didrik Hoiseth A, et al. The prognostic value of measurement of high-sensitive cardiac troponin $\mathrm{T}$ for mortality in a cohort of stable chronic obstructive pulmonary disease patients. BMC Pulm Med 2016; 16: 164 .

11 Adamson PD, Anderson JA, Brook RD, et al. Cardiac troponin I and cardiovascular risk in patients with chronic obstructive pulmonary disease. J Am Coll Cardiol 2018; 72: 1126-1137.

12 Ye C, Younus A, Malik R, et al. Subclinical cardiovascular disease in patients with chronic obstructive pulmonary disease: a systematic review. QJM 2017; 110: 341-349.

13 Rubin J, Matsushita K, Lazo M, et al. Determinants of minimal elevation in high-sensitivity cardiac troponin $\mathrm{T}$ in the general population. Clin Biochem 2016; 49: 657-662.

14 Agusti A, Noell G, Brugada J, et al. Lung function in early adulthood and health in later life: a transgenerationa cohort analysis. Lancet Respir Med 2017; 5: 935-945.

15 Nadruz W J, Goncalves A, Claggett B, et al. Influence of cigarette smoking on cardiac biomarkers: the Atherosclerosis Risk in Communities (ARIC) Study. Eur I Heart Fail 2016; 18: 629-637.

16 Criner GJ, Connett JE, Aaron SD, et al. Simvastatin for the prevention of exacerbations in moderate-to-severe COPD. N Engl J Med 2014; 370: 2201-2210.

17 Dransfield MT, Voelker H, Bhatt SP, et al. Metoprolol for the prevention of acute exacerbations of COPD. N Engl J Med 2019; 381: 2304-2314.

18 Franssen FME, Vanfleteren LEGW. Differential diagnosis and impact of cardiovascular comorbidities and pulmonary embolism during COPD exacerbations. In: Burgel P, Contoli M, López-Campos JL, eds. Acute exacerbations of pulmonary diseases (ERS Monograph). Sheffield, European Respiratory Society, 2017; pp. 114-128.

19 Hurst JR, Vestbo J, Anzueto A, et al. Susceptibility to exacerbation in chronic obstructive pulmonary disease. N Engl J Med 2010; 363: 1128-1138. 
20 Kunisaki KM, Dransfield MT, Anderson JA, et al. Exacerbations of chronic obstructive pulmonary disease and cardiac events. a post hoc cohort analysis from the SUMMIT randomized clinical trial. Am J Respir Crit Care Med 2018; 198: 51-57.

21 Pavasini R, Tavazzi G, Biscaglia S, et al. Amino terminal pro brain natriuretic peptide predicts all-cause mortality in patients with chronic obstructive pulmonary disease: systematic review and meta-analysis. Chron Respir Dis 2017; 14: 117-126.

22 Romundstad S, Naustdal T, Romundstad PR, et al. COPD and microalbuminuria, a 12-year follow-up study. Eur Respir J 2014; 43: 1042-1050.

23 Kistorp C, Raymond D, Pedersen F, et al. N-terminal pro-brain natriuretic peptide C-reactive protein, and urinary albumin levels as predictors of mortality and cardiovascular events in older adults. JAMA 2005; 239: 1609-1616.

24 Lindberg A, Bjerg A, Rönmark E, et al. Prevalence and underdiagnosis of COPD by disease severity and the attributable fraction of smoking. Report from the Obstructive Lung Disease in Northern Sweden Studies. Respir Med 2006; 100: 264-272. 